\title{
HOLOMORPHIC LINE BUNDLES OVER HILBERT FLAG VARIETIES
}

\author{
A.G. HELMINCK AND G.F. HELMINCK
}

\begin{abstract}
Abst ract. In this contribution we present a geometric realization of an infinite dimensional analogue of the irreducible representations of the unitary group. This requires a detailed analysis of the structure of the flag varieties involved and the line bundles over it. These constructions are of importance in quantum field theory and in the framework of integrable systems. As an application, it is shown how they occur in the latter context.
\end{abstract}

\section{Int roduct ion}

It is a classical result that the irreducible representations of the unitary group $U(H)$ of a finite dimensional space $H$ can be realized geometrically as the natural action of $U(H)$ on the holomorphic sections of a holomorphic line bundle over a space of flags in $H$. Infinite dimensional analogues of some of these representations occur in quantum field theory, see e.g. [2]. In this paper we consider a separable Hilbert space $H$ and we introduce a manifold of flags in $H$ over which there exist holomorphic line bundles that are similar to the finite dimensional ones. The definition of the flag variety and some of its properties can be found in the first section. The second section is devoted to the construction of the holomorphic line bundles and the analysis of their space of holomorphic sections. As an application, we show in the final section what role the geometry plays in the context of some integrable systems. A more detailed description of the content of the different sections is as follows.

The first subsection starts with giving the type of flags in $H$ that will be considered. Here the basic flag $F^{(0)}$ serves as a model. The flag variety $\mathfrak{F}$ is a homogeneous space for a certain unitary group $U_{\text {res }}(H)$. As in the finite dimensional case one can see $\mathfrak{\Im}$ also as a homogeneous space for a larger group of automorphisms of $H$, namely $G l_{\text {res }}(H)$. This is the analogue of the general linear group in this framework. The second subsection is devoted to an explicit description of the manifold structure on $\mathfrak{F}$. The first difference with the finite dimensional case appears at the description of the connected components of $\mathfrak{F}$ in the third subsection.

After these general considerations we start the new section with the technical prerequisites for the construction of the holomorphic line bundles. First we choose

1991 Mathematics Subject Classification. 22E65, 14M15, 35Q58,43A80, 17B65. 
a suitable orthonormal basis of $H$, we order its index set $S$ conveniently and introduce the Weylgroup $W$ of $U_{\text {res }}(H)$. Next we show that the charts around the points in the $W$-orbit through $F^{(0)}$ cover $\mathfrak{\Im}$. Let $\mathfrak{F}^{(0)}$ be the connected component of $\mathfrak{F}$ that contains $F^{(0)}$. The foregoing results enable you to introduce a dense subspace $\mathfrak{F}(\infty)$ of $\mathfrak{F}^{(0)}$ that is the direct limit of finite dimensional flag varieties. The space $\mathfrak{f}^{(0)}$ can be written as a homogeneous manifold $G_{2} / \mathfrak{T}$, where $G_{2}$ is a suitable Banach Lie group for which certain subdeterminants are defined. To come to the form of the group $G_{2}$ one has to introduce the notion of an admissible permutation of the index set $S$. Then we have the necessary ingredients for the introduction of a collection of holomorphic line bundles over $\mathfrak{F}^{(0)}$ in the subsequent subsection. Let $G l_{\text {res }}^{(0)}(H)$ be the connected component of $G l_{\text {res }}(H)$. If one wants to lift the $G l_{\text {res }}^{(0)}(H)$-action to these line bundles, one is forced to introduce a central extension $\widetilde{G}$ of the group $G l_{\text {res }}^{(0)}(H)$. We conclude this section with a subsection describing the space of holomorphic sections of these bundles and the irreducibility of the $\widetilde{G}$-action on it.

The third section starts with giving a framework for the equations of the multicomponent KP-hierarchy. Next we give the geometric ingredients that are needed for the construction of solutions of the equations from the first subsection. In the final subsection we give a geometric construction of solutions of the multicomponent KP-hierarchy and a geometric interpretation of these non-linear equations and some other related systems.

\section{Properties of Hil ber $t$ fl ag manifol ds}

2.1. The flag variety. Let $H$ be a separable complex Hilbert space with innerproduct $<\cdot, \cdot\rangle$. We will consider certain chains of subspaces in $H$ and as in the finite dimensional case one has to specify the "size" of the components. Therefore we start with an orthogonal decomposition of $H$,

$$
H=H_{1} \oplus \ldots \oplus H_{m}, \quad \text { where } H_{i} \perp H_{j} \text { for } i \neq j .
$$

We assume that $m_{i}=\operatorname{dim}\left(H_{i}\right)$ satisfies $1 \leq m_{i} \leq \infty$. Let $p_{i}, 1 \leq i \leq m$, be the orthogonal projection of $H$ onto $H_{i}$. Then we will use throughout this paper the following

Notation 2.1.1. If $g$ belongs to $B(H)$, the space of bounded linear operators from $H$ to $H$, then $g=\left(g_{i j}\right), 1 \leq i \leq m$ and $1 \leq j \leq m$, denotes the decomposition of $g$ w.r.t. the $\left\{H_{i} \mid 1 \leq i \leq m\right\}$. That is to say $g_{i j}=p_{i} \circ g \mid H_{j}$.

To the decomposition (1) we associate the basic flag $F^{(0)}$ given by

$$
0 \subset H_{1} \subset \ldots \subset \bigoplus_{j=1}^{r} H_{j} \subset \ldots \subset H .
$$


Now we consider in $H$ flags $F=\{F(0), \ldots, F(m)\}$, that is to say chains of closed subspaces of $H$,

$$
\{0\}=F(0) \subset F(1) \subset \ldots \subset F(m)=H,
$$

that are comparable to the basic flag $F^{(0)}$, i.e. for all $i, 1 \leq i \leq m$,

$$
\operatorname{dim}(F(i) / F(i-1))=\operatorname{dim}\left(H_{i}\right) .
$$

To such a flag $F$ is associated an orthogonal decomposition of $H$,

$$
H=F_{1} \oplus \ldots \oplus F_{m}, \quad \text { where } F_{i}=F(i) \cap F(i-1)^{\perp} .
$$

We will denote a flag $F$ by $F=\{F(0), \ldots, F(m)\}$ as well as $F=\left\{F_{1}, \ldots, F_{m}\right\}$.

The class of flags one obtains in this way is still too wide and we will require that our flags do not differ too much from the basic flag. The condition by which we express this property is a natural generalization of that used in [20] for the Grassmann manifold and is such that it permits you to construct suitable measures on your manifold, see [8] and [19]. Moreover the connected component $\mathfrak{F}^{(0)}$ of $\mathfrak{F}$, containing $F^{(0)}$, is a homogeneous space for the group of all invertible operators of the form identity + a Hilbert-Schmidt operator and this group comes up in a natural way in quantum field theory, see [22] and [3]. There are, however, situations where other spaces of compact operators have to be considered, see e.g. [18].

Definition 2.1.2. Let $\mathfrak{F}$ be the collection of flags $F=\left\{F_{1}, \ldots, F_{m}\right\}$, satisfying $\operatorname{dim}\left(F_{i}\right)=\operatorname{dim}\left(H_{i}\right)$, such that for all $i$ and $j$ with $j \neq i$, the orthogonal projection $p_{j}: F_{i} \rightarrow H_{j}$ is a Hilbert-Schmidt operator. We call $\mathfrak{\wp}$ the flag variety corresponding to the decomposition (1).

Example 2.1.3. The Grassmann manifold $G r(H)$ that plays an important role in [20] and [21] corresponds to the case that $H$ is the space of powerseries

$$
\begin{aligned}
H & =L^{2}\left(S^{1}, \mathbb{C}\right)=\left\{\sum_{n \in \mathbb{Z}} a_{n} z^{n}, a_{n} \in \mathbb{C}, \sum_{n \in \mathbb{Z}}\left|a_{n}\right|^{2}<\infty\right\}, \\
H_{1} & =\left\{\sum_{n \geq 0} a_{n} z^{n} \in H\right\} \text { and } H_{2}=\left\{\sum_{n<0} a_{n} z^{n} \in H\right\} .
\end{aligned}
$$

For a decomposition that plays a role in quantum field theory, see [2].

As in the finite dimensional case, the space $\mathfrak{F}$ is a homogeneous space for a certain unitary group. Let $F=\left\{F_{1}, \ldots, F_{m}\right\}$ belong to $\mathfrak{\wp}$. From the definition of $\mathfrak{\wp}$ we know that there is for each $i, 1 \leq i \leq m$, an isometry $u_{i}$ between $H_{i}$ and $F_{i}$. If we put $u=u_{1} \oplus \ldots \oplus u_{m}$, then $u$ belongs to the group of unitary transformations, $U(H)$, of $H$ and for each $1 \leq i \leq m$ we have

$$
u\left(\bigoplus_{j=1}^{i} H_{j}\right)=\bigoplus_{j=1}^{i} F_{j} .
$$


In other words, the flag $F$ is the image under $u$ of the basic flag. The condition defining $\mathfrak{F}$ implies that $u=\left(u_{i j}\right)$ satisfies: $u_{i j}$ is a Hilbert-Schmidt operator for $i \neq j$. This brings us to the introduction of the following group.

Definition 2.1.4. The restricted unitary group, $U_{\text {res }}(H)$, consists of all $u=\left(u_{i j}\right)$ in $U(H)$ such that $u_{i j}$ is a Hilbert-Schmidt operator if $i \neq j$.

The group $U_{\text {res }}(H)$ is defined in such a way that, reversibly, for each $g \in U_{\text {res }}(H)$ the flag $g\left(F^{(0)}\right)$ belongs to $\mathfrak{r}$. If we extend a unitary transformation $\sigma$ of $H_{i}$ in the trivial way to one of $H$, then this extended transformation belongs to $U_{\text {res }}(H)$. Thus we have a natural embedding of each $U\left(H_{i}\right)$ into $U_{\text {res }}(H)$. Clearly the stabilizer of $F^{(0)}$ in $U_{\text {res }}(H)$ is equal to $\prod_{i=1}^{m} U\left(H_{i}\right)$ and therefore we can identify $\mathfrak{\mho}$ with the homogeneous space

$$
U_{\mathrm{res}}(H) / \prod_{i=1}^{m} U\left(H_{i}\right) .
$$

For several reasons, like the description of the manifold structure on $\mathfrak{\mho}$ and the consideration of non-unitary flows on $\mathfrak{F}$, it is convenient to have a description of $\mathfrak{F}$ as the homogeneous space of a larger group of automorphisms of $H$. The Banach structure of this group follows directly from that of its Lie algebra. Therefore we start with the analogue of the Lie algebra of the general linear group.

Definition 2.1.5. A restricted endomorphism of $H$ is a $u=\left(u_{i j}\right)$ in $B(H)$ such that $u_{i j}$ is a Hilbert-Schmidt operator for all $i \neq j$. We denote the space of all restricted endomorphisms of $H$ by $B_{\text {res }}(H)$.

The space $B_{\text {res }}(H)$ is a subalgebra of $B(H)$ since the collection of HilbertSchmidt operators is closed under left and right multiplication with bounded operators. Hence it is also a Lie subalgebra of the Lie algebra $B(H)$. On $B_{\text {res }}(H)$ we will introduce a norm. This requires some general notations.

Notation 2.1.6. If $K_{1}$ and $K_{2}$ are Hilbert spaces, then we denote the space of HilbertSchmidt operators from $K_{1}$ to $K_{2}$ by $\mathfrak{I}_{2}\left(K_{1}, K_{2}\right)$ and the Hilbert-Schmidt norm by $\|\cdot\|_{H S}$. If $K_{2}$ is equal to $K_{1}$, then we simply write $\mathfrak{I}_{2}\left(K_{1}\right)$ instead of $\mathfrak{I}_{2}\left(K_{1}, K_{1}\right)$.

For all $i$ and $j$, we extend the elements of $\mathfrak{I}_{2}\left(H_{i}, H_{j}\right)$ outside $H_{i}$ by zero and obtain thus a natural embedding of $\mathfrak{I}_{2}\left(H_{i}, H_{j}\right)$ into $\mathfrak{I}_{2}(H)$. The algebra $B_{\text {res }}(H)$ becomes a Banach algebra if we equip it with the norm $\|\cdot\|_{2}$ defined by

$$
\|u\|_{2}=\|u\|+\sum_{i \neq j}\left\|u_{i j}\right\|_{H S}
$$

If $G l(H)$ denotes the group of invertible elements in $B(H)$, then we consider 
Definition 2.1.7. The restricted linear group, $G l_{\mathrm{res}}(H)$, consists of $\{g \mid g \in$ $\left.G l(H) \cap B_{\text {res }}(H)\right\}$.

One easily verifies that $G l_{\text {res }}(H)$ consists of the invertible elements in $B_{\text {res }}(H)$ and, as such, has a natural Banach manifold structure. With each $g$ in $G l_{\text {res }}(H)$ we can associate the flag

$$
0 \subset g H_{1} \subset g\left(H_{1} \oplus H_{2}\right) \subset \ldots g\left(H_{1} \oplus \ldots \oplus H_{i}\right) \subset \ldots \subset H .
$$

From the definition of $G l_{\text {res }}(H)$ one sees directly that this flag belongs to $\mathfrak{F}$. The stabilizer in $G l_{\text {res }}(H)$ of the basic flag is the "parabolic subgroup"

$$
P=\left\{g \mid g \in G l_{\text {res }}(H), g=\left(\begin{array}{cccc}
g_{11} & \ldots & \ldots & g_{1 m} \\
0 & \ddots & & \vdots \\
\vdots & \ddots & \ddots & \vdots \\
0 & \ldots & 0 & g_{m m}
\end{array}\right) \text {, with } g_{i i} \in G l\left(H_{i}\right)\right\} \text {. }
$$

Thus we can identify $\mathfrak{\wp}$ also with the homogeneous space $G l_{\text {res }}(H) / P$. Let $\tau$ : $G l_{\text {res }}(H) \rightarrow \mathfrak{F}$ be the projection $\tau(g)=g \cdot F^{(0)}$. On $\mathfrak{\Im}$ we put the quotient topology that makes $\tau$ into an open continuous map. In the next subsection we will put a Hilbert manifold structure on $\mathfrak{r}$.

2.2. The manifold structure of $\mathfrak{\wp}$. To get an idea how the space $\mathfrak{\wp}$ locally looks like near the basic flag, we consider the open set $\Omega$ in $G l_{\text {res }}(H)$ given by

$$
\Omega=\left\{g \in G l_{\mathrm{res}}(H) \mid\left(\begin{array}{ccc}
g_{11} & \ldots & g_{1 i} \\
\vdots & & \vdots \\
g_{i 1} & \ldots & g_{i i}
\end{array}\right) \in G l_{\mathrm{res}}\left(H_{1} \oplus \ldots \oplus H_{i}\right) \quad \text { for all } 1 \leq i \leq m\right\} .
$$

As in the finite dimensional case, $\Omega$ is called the "big cell". Let $U_{-}$be the subgroup of $G l_{\text {res }}(H)$ defined by

$$
U_{-}=\left\{\begin{array}{l|l}
g=\left(g_{i j}\right) \in G l_{\mathrm{res}}(H) & \begin{array}{l}
g_{i i}=I d_{H_{i}} \text { for all } i \\
g_{i j}=0 \text { for } j>i
\end{array}
\end{array}\right\}
$$

The groups $P$ and $U_{-}$are given the topology induced by that of $G l_{\mathrm{res}}(H)$. By induction on the number of components of the decomposition of $H$, one proves then

Lemma 2.2.1. The map $(u, p) \mapsto u p$ from $U_{-} \times P \rightarrow G l_{\text {res }}(H)$ determines $a$ homeomorphism between $U_{-} \times P$ and $\Omega$.

In particular we see that the restriction of $\tau$ to $U_{-}$gives you a homeomorphism $u \mapsto u F^{(0)}$ between $U_{-}$and the open neighborhood $\tau(\Omega)$ of $F^{(0)}$. Clearly the group $U_{-}$is homeomorphic to the Hilbert space $\left(E,\|\cdot\|_{H S}\right)$ with

$$
E=\bigoplus_{\substack{1 \leq j \leq m-1 \\ i>j}} \mathfrak{I}_{2}\left(H_{j}, H_{i}\right)
$$


Around each point of $\mathfrak{\wp}$, we will give a neighborhood homeomorphic to $E$. Note that from the definition of $\Omega$ one can conclude directly that

$\tau(\Omega)=\left\{F=\left(F_{i}\right) \in \mathfrak{\Im} \mid \bigoplus_{j \leq l} p_{j}: \bigoplus_{j \leq l} F_{j} \rightarrow \bigoplus_{j \leq l} H_{j} \quad\right.$ is a bijection for all $\left.1 \leq l \leq m\right\}$.

This characterization of $\tau(\Omega)$ tells you what to choose around a general point of $\mathfrak{\wp}$.

This requires, however, the introduction of a

Notation 2.2.2. If $W$ is closed subspace of $H$, then we denote the orthogonal projection on $W$ by $p_{W}$.

Consider a $F=\left(F_{1}, \ldots, F_{m}\right)$ in $\mathfrak{\Im}$. Then the analogue of $\tau(\Omega)$ for $F$ is

$U_{F}=\left\{V=\left(V_{i}\right)\right.$ in $\widetilde{\mho} \mid \bigoplus_{i \leq l} p_{F_{i}}: \bigoplus_{i \leq l} V_{i} \rightarrow \bigoplus_{i \leq l} F_{i} \quad$ is a bijection for all $\left.1 \leq l \leq m\right\}$.

It is convenient to have a special expression for the flags in $U_{F}$.

Definition 2.2.3. A flag $V$ in $U_{F}$ is called transversal to $F$.

Let $g_{F}$ be an element of $U_{\text {res }}(H)$ such that $g_{F} \cdot F^{(0)}=F$. Instead of the big cell $\Omega$ in $G l_{\text {res }}(H)$ with respect to the decomposition $H=H_{1} \oplus \ldots \oplus H_{m}$, we could also have introduced a big cell with respect to $H=F_{1} \oplus \ldots \oplus F_{m}$ and it will be clear that this set can be written as

$$
g_{F} U_{-} P\left(g_{F}^{-1}\right) .
$$

Consequently, we get for $U_{F}$ that

$$
U_{F}=\left\{g_{F} u p\left(g_{F}\right)^{-1} F \mid \text { with } u \in U_{-} \text {and } p \in P\right\}=\tau\left(g_{F} U_{-} P\right) .
$$

Assume that we have chosen for each $F$ in $\mathfrak{f}$ a $g_{F}$ in $U_{\text {res }}(H)$ with $g_{F} \cdot F^{(0)}=F$. Then we can define for each $F$ in $\mathfrak{\wp}$ a homeomorphism $\varphi_{F}: U_{F} \rightarrow E$ by

$$
\varphi_{F}\left(g_{F} u F^{(0)}\right)=u-I d .
$$

The main result of this section is

Proposition 2.2.4. The $\left(U_{F}, \varphi_{F}\right)$ are the charts of an analytic E-manifold structure on $\mathfrak{乛}$.

Proof. It is sufficient to show for each $U_{F^{(1)}}$ and $U_{F^{(2)}}$ with $U_{F^{(1)}} \cap U_{F^{(2)}} \neq$ that

$$
\varphi_{F^{(2)}} \circ \varphi_{F^{(1)}}^{-1}: \varphi_{F^{(1)}}\left(U_{F^{(1)}} \cap U_{F^{(2)}}\right) \rightarrow \varphi_{F^{(2)}}\left(U_{F^{(1)}} \cap U_{F^{(2)}}\right)
$$

is an analytic map. This follows from how the $U_{-}$-component of $\left(g_{F^{(2)}}\right)^{-1} g_{F^{(1)}} u$ actually depends on $u$. This is given by Lemma 2.2.1.

It will be clear from the construction that the group $G l_{\text {res }}(H)$ acts analytically on the manifold $\mathfrak{\wp}$. 
2.3. The connected components of $\boldsymbol{G} \boldsymbol{l}_{\text {res }}(\boldsymbol{H})$. Let $g=\left(g_{i j}\right)$ be an element of $G l_{\text {res }}(H)$ and put $g^{-1}=\left(h_{i j}\right)$. Then we have, by definition for all $i, 1 \leq i \leq m$,

$$
g_{i i} h_{i i}=I d_{H_{i}}-\sum_{k \neq i} g_{i k} h_{k i} \text {. }
$$

This implies that each $g_{i i}$ is a Fredholm operator, that is to say it has a finite dimensional kernel and cokernel. The collection of Fredholm operators on a Hilbert space $K$ is denoted by $\Phi(K)$ and it is an open part of the space $B(K)$. Its connected components are given by the index, which is defined as

$$
\operatorname{ind}(B)=\operatorname{dim}(\operatorname{ker}(B))-\operatorname{dim}(\operatorname{coker}(B)), \quad \text { for } B \in \Phi(K) \text {. }
$$

Since all off-diagonal operators are Hilbert-Schmidt and hence compact, the operator

$$
\tilde{g}=\left(\begin{array}{ccc}
g_{11} & & 0 \\
& \ddots & \\
0 & & g_{m m}
\end{array}\right), \quad \text { where } g=\left(g_{i j}\right) \in G l_{\text {res }}(H),
$$

is a Fredholm operator of index zero. Hence we have that the indices of the $\left\{g_{i i} \mid\right.$ $1 \leq i \leq m\}$ satisfy

$$
\sum_{i=1}^{m} \operatorname{ind}\left(g_{i i}\right)=0 \quad \text { and } \quad \operatorname{ind}\left(g_{k k}\right)=0 \quad \text { if } \quad m_{k}<\infty .
$$

These relations lead to the introduction of the subgroup $Z$ of $\mathbb{Z}^{m}$ defined by

$$
Z=\left\{z=\left(z_{i}\right) \in \mathbb{Z}^{m} \mid \sum_{i=1}^{m} z_{i}=0, z_{k}=0 \quad \text { if } \quad m_{k}<\infty\right\} .
$$

The standard properties of the index imply that the map $\underline{i}: G l_{\text {res }}(H) \rightarrow Z$,

$$
\left.g \mapsto \operatorname{ind}\left(g_{11}\right), \ldots, \operatorname{ind}\left(g_{m m}\right)\right),
$$

is a continuous grouphomomorphism. Hence the sets

$$
G l_{\text {res }}^{(z)}(H)=\left\{g \mid g \in G l_{\text {res }}(H), \underline{i}(g)=z\right\}, \quad \text { with } z \in Z,
$$

are open. In fact, they are exactly the connected components of $G l_{\text {res }}(H)$, for

Proposition 2.3.1. For each $z \in Z$, the set $G l_{\text {res }}^{(z)}(H)$ is non-empty and connected.

Proof. Let $z=\left(z_{i}\right)$ be in $Z$ and let $h_{i} \in \Phi\left(H_{i}\right)$ be such that ind $\left(h_{i}\right)=z_{i}$. Then

$$
h=\left(\begin{array}{ccc}
h_{1} & & 0 \\
& \ddots & \\
0 & & h_{m}
\end{array}\right)
$$

belongs to $\Phi(H)$ and has index zero. Therefore one can add to $h$ an isomorphism between the kernel of $h$ and the orthogonal complement of the image of $h$ to obtain an element of $G l_{\text {res }}^{(z)}(H)$. The connectivity is proven in two steps: first one notes 
that $P$ is connected, since all the $G l\left(H_{i}\right)$ are connected, see [17]. One concludes by showing that every element of $G l_{\text {res }}^{(0)}(H)$ can be joined by a continuous path with an element of $P$.

Since $P$ is connected, we see that

Corollary 2.3.2. The connected components of $\mathfrak{\wp}$ are given by

$$
\mathfrak{\Im}^{(z)}=\left\{g \cdot F^{(0)} \mid g \in G l_{\text {res }}^{(z)}(H)\right\} .
$$

Remark 2.3.3. The space $\mathfrak{\wp}$ possesses many more properties reminiscent of the finite dimensional flag varieties, like a Kähler structure and a stratification. They are not treated here but can be found in [9] and [11]. In [9] one finds also the Birkhoff decomposition for the group $G l_{\text {res }}(H)$.

Remark 2.3.4. A holomorphic line bundle $L$ over $\mathfrak{F}$ consists simply of a collection of holomorphic line bundles $\left\{L_{z} \rightarrow \mathfrak{F}^{(z)} \mid z \in Z\right\}$. Therefore we restrict our attention to line bundles over $\mathfrak{\Im}^{(0)}$.

\section{Hol omorphic 1 ine bundles over $\mathfrak{F}^{(0)}$}

3.1. A special covering of $\mathfrak{\wp}$. In this subsection we choose a suitable orthonormal basis of $H$ and we introduce a collection of charts of $\mathfrak{F}$ that can be described completely in terms of the index set of this orthonormal basis. In particular these charts cover $\mathfrak{F}$ and enable you to give a combinatorial description of the Birkhoff decomposition of $G l_{\text {res }}(H)$ and to construct concretely a collection of holomorphic line bundles over $\mathfrak{F}^{(0)}$.

Let $\left\{e_{s} \mid s \in S_{i}\right\}$ be an orthonormal basis of $H_{i}$ for all $i, 1 \leq i \leq m$. We will put a total order on each $S_{i}$ and we will combine them to one on $S=\bigcup_{1 \leq i \leq m} S_{i}$ by requiring that $s_{j}<s_{i}$ for all $s_{i} \in S_{i}$ and $s_{j} \in S_{j}$ with $j>i$. Since $H$ is separable we can choose for

$$
S_{1}=\left\{k \in \mathbb{Z} \mid 0 \leq k<m_{1}\right\} \quad \text { and for } \quad S_{2}=\left\{k \in \mathbb{Z} \mid 0>k>m_{2}-1\right\} .
$$

The usual order on $\mathbb{Z}$ induces one on $S_{1}$ and $S_{2}$ that satisfies the requirement above. We would like to continue in this fashion, but $\mathbb{Z}$ might already be exhausted. Therefore we take for $S_{j}, j \geq 3$, a suggestive description as a collection of formal numbers

$$
S_{j}=\left\{-m_{2} \ldots-m_{j-1}-k, \quad \text { with } \quad k \in \mathbb{N}, 1 \leq k<m_{j}+1\right\} .
$$

The set $S_{j}$ is ordered in the following way

$$
-m_{2} \ldots-m_{j-1}-k \geq-m_{2} \ldots-m_{j-1}-\ell \Leftrightarrow-k \geq-\ell .
$$

Now that we have an orthonormal basis $\left\{e_{s} \mid s \in S\right\}$ of $H$ with a totally ordered index set $S$, we can associate to each bounded $g$ in $B(H)$ an $S \times S$-matrix $[g]$ with coefficients

$$
g_{s t}=<g\left(e_{t}\right), e_{s}>, \quad \text { where } s \text { and } t \text { are in } S .
$$


Several properties of $g$ can be described in terms of these matrixcoefficients. For example, the operator $g$ in $B(H)$ belongs to $B_{\text {res }}(H)$ if and only if

$$
\sum_{\substack{t \in S_{j} \\ j \neq i}} \sum_{\substack{s \in S_{i} \\ 1 \leq i \leq m}}\left|g_{s t}\right|^{2}<\infty .
$$

Remark 3.1.1. Recall that the Lie algebra $A_{\infty}$, see [15], can be realized as a central extension of the Lie algebra $\overline{g l}_{\infty}$ of $\mathbb{Z} \times \mathbb{Z}$-matrices $\left\{a_{i j}\right\}$ of finite width matrices, i.e. those matrices $\left\{a_{i j}\right\}$ satisfying $a_{i j}=0$ if $|i-j|>N$ for some $N \in \mathbb{N}$. The Lie algebra of the matrices of the elements of $B_{\text {res }}(H)$ can thus been seen as a completion of a bounded version of $\overline{g l}_{\infty}$. Also the central extension $A_{\infty}$ of $\overline{g l}_{\infty}$ occurs in a natural way in this geometric framework, see the subsection 3.3.

A type of operator that we will meet in the sequel is

Definition 3.1.2. An operator $g$ in $B(H)$ is called a "finite-size" operator if it has only a finite number of non-zero matrixcoefficients w.r.t. the $\left\{e_{s} \mid s \in S\right\}$.

Remark 3.1.3. By the choice of the order on $S$, the columns of $[g]$ increase from "east" to "west" in the numbering and the rows of $[g]$ from "south" to "north". This is different from the finite dimensional habits, but is in agreement with that in [20].

In the sequel we will frequently use some notations related to subsets of $S$.

Notation 3.1.4. The number of elements in a subset $A$ of $S$ is denoted by \#A.

Notation 3.1.5. If $A$ is a non-empty subset of $S$, then we denote the closure of the span of the $\left\{e_{s} \mid s \in A\right\}$ by $H_{A}$. If $A$ is empty, then $H_{A}$ denotes the space $\{0\}$. It is convenient to denote the orthogonal projection onto $H_{A}$ by $p_{A}$.

Maps between subsets of $S$ have a direct translation to partial isometries between closed subspaces of $H$, i.e.

Notation 3.1.6. If $A$ and $B$ are subsets of $S$ and $\tau: A \rightarrow B$ is some map, then we denote by $\underline{\tau}$ the mapping from $H_{A}$ to $H_{B}$ given by

$$
\underline{\tau}\left(\sum_{t \in A} \lambda_{t} e_{t}\right)=\sum_{t \in A} \lambda_{t} e_{\tau(t)} .
$$

Now that we have chosen the orthonormal basis $\left\{e_{s} \mid s \in S\right\}$, we can introduce "diagonaloperators" in $B_{\text {res }}(H)$. Suppose that we have a set of bounded complex numbers

$$
\left\{\delta_{s} \mid s \in S, \delta_{s} \in \mathbb{C} \text { and }\left|\delta_{s}\right| \leq M \text { for all } s \in S\right\}
$$


Then we can associate to it a diagonal operator $\operatorname{diag}\left(\delta_{S}\right)$ in $B(H)$ by

$$
\operatorname{diag}\left(\delta_{S}\right)\left(\sum_{t \in S} \lambda_{t} e_{t}\right)=\sum_{t \in S} \delta_{t} \lambda_{t} e_{t} .
$$

Inside $G l_{\text {res }}(H)$ we have then the "maximal torus"

$$
T=\left\{g \mid g \in G l_{\text {res }}(H), g=\operatorname{diag}\left(\delta_{s}\right)\right\} .
$$

Clearly $T$ is commutative and with the help of the matrix one shows that the centralizer of $T$ inside $G l(H)$ is equal to $T$. Hence we have

Lemma 3.1.7. The centralizer $Z(T)$ of $T$ in $G l_{\text {res }}(H)$ is equal to $T$.

Each permutation $\sigma$ of $S$ determines a unitary map $\underline{\sigma}: H \rightarrow H$ as in (3.1.5). With the help of the matrix, one shows that the normalizer of $T$ in $G l(H)$ consists of

$$
\{t \cdot \underline{\sigma} \mid t \in T, \sigma \text { a permutation of } S\} .
$$

Hence, if we define the the subgroup $W$ of $U_{\text {res }}(H)$ as

$$
W=\left\{\underline{\sigma} \mid \underline{\sigma} \in U_{\text {res }}(H), \sigma \text { a permutation of } S\right\},
$$

then we have

Corollary 3.1.8. The normalizer $N(T)$ of $T$ in $G l_{\text {res }}(H)$ is the semi-direct product of $W$ and $T$. In particular, we see that $W$ is isomorphic to $N(T) / Z(T)$ and we call $W$ the Weylgroup of $T$.

To each $\underline{\sigma}$ in $W$, corresponds a partition $\Sigma=\bigcup_{i \geq 1} \Sigma_{i}$, where $\Sigma_{i}=\sigma\left(S_{i}\right)$. The concrete description of which partitions occur in this way, brings one in a natural way to the consideration of subsets of $S$ that are "equal up to a finite set". Therefore we define

Definition 3.1.9. If $A$ and $B$ are subsets of $S$, then we call $A$ and $B$ commensurable (notation $A \approx B$ ) if $A-\{A \cap B\}$ and $B-\{A \cap B\}$ are finite. We write $i(A, B)$ for the number

$$
\#\{A-\{A \cap B\}\}-\#\{B-\{A \cap B\}\} .
$$

This commensurability is equivalent to: the orthogonal projection $p_{B}: H_{A} \rightarrow H_{B}$ is a Fredholm operator with index $i(A, B)$. Let $\Sigma=\left\{\Sigma_{i} \mid i \leq i \leq m\right\}$ be an arbitrary partition of $S$ into $m$ disjoint parts. Then this partition corresponds to an element of $W$, if and only if the following two conditions hold:

$$
\begin{aligned}
& \# \Sigma_{i}=\# S_{i} \quad \text { for all } i, 1 \leq i \leq m \text {, and } \\
& \Sigma_{i} \approx S_{i} \quad \text { for all } i, 1 \leq i \leq m
\end{aligned}
$$

To any partition $\Sigma$ satisfying these conditions there corresponds a flag $F_{\Sigma}$ in $\mathfrak{f}$ given by

$$
\circ \subset H_{\Sigma_{1}} \subset H_{\Sigma_{1} \cup \Sigma_{2}} \subset \ldots \subset H_{S}=H .
$$


In the sequel we will frequently make use of the following notion

Definition 3.1.10. An element in $H$ is said to be of order $s, s \in S$, if it has the form

$$
h=a_{s} e_{s}+\sum_{\substack{t \in S \\ t<s}} a_{t} e_{t}, \quad \text { with } a_{s} \neq 0,
$$

The union of all the elements of some order $s$ in $S$ and $\{0\}$ is called the space of elements of finite order.

For each $z$ in $Z$, we denote the collection of partition $\Sigma$ of $S$ such that $F_{\Sigma}$ belongs to $\mathfrak{F}^{(z)}$, by $\widetilde{\subseteq}(z)$. The basic property of the $\left\{F_{\Sigma} \mid \Sigma \in \subseteq(z)\right\}$ is

Proposition 3.1.11. For each flag $F=(F(1), \ldots, F(m))$ in $\mathfrak{F}^{(z)}$, there is a $\Sigma$ in $\Im(z)$ such that $F$ is transversal to $F_{\Sigma}$.

Proof. Let $g \in G l_{\text {res }}(H)$ be such that $F=g . F^{(0)}$. First we show that there is a $\Sigma_{1}$, commensurable with $S_{1}$ and with $\# S_{1}=\# \Sigma_{1}$, such that $\left.p_{\Sigma_{1}} \circ g\right|_{H_{1}}$ is an isomorphism between $H_{1}$ and $H_{\Sigma_{1}}$. Since $p_{1}\left(g\left(H_{1}\right)\right)$ has finite codimension in $H_{1}$, we can find a $S_{1}(n)=\left\{k \mid k \in S_{1}, k \geq n\right\}, n \geq 0$, such that $F(1)=g\left(H_{1}\right)$ projects surjectively onto $H_{S_{1}(n)}$. The kernel of this projection has a basis $\left\{h_{j} \mid 1 \leq j \leq N\right\}$ of elements of finite order, i.e.

$$
h_{j}=e_{s_{j}}+\sum_{\substack{t \in S \\ t<s_{j}}} \alpha_{j}(t) e_{t} \quad, \quad \text { where } s_{i} \neq s_{j} \text { for } i \neq j .
$$

It is clear that we can take $\Sigma_{1}=S_{1}(n) \cup\left\{s_{j} \mid 1 \leq j \leq N\right\}$. The other parts of the desired partition $\Sigma$ of $S$ are constructed step by step form $\Sigma_{1}$. For, assume that we have found disjoint $\left\{\Sigma_{j} \mid j \leq i\right\}$ with $\Sigma_{j} \approx S_{j}$ and $\# S_{j}=\# \Sigma_{j}$ such that the orthogonal projection of $F(j)$ onto $\bigoplus_{\ell \leq j} H_{\ell}$ is a bijection for all $j \leq i$. Then we know that $p_{i+1}\left(g\left(H_{i+1}\right)\right)$ has finite codimension in $H_{i+1}$. So there exists a subset $\widetilde{S}_{i+1}$ of $S_{i+1}$, commensurable with $S_{i+1}$ and disjoint of $\Sigma_{1} \cup \ldots \Sigma_{i}$, such that $F(i+1)$ projects surjectively onto $H_{\Sigma_{1}} \oplus \ldots \oplus H_{\Sigma_{i}} \oplus H_{\widetilde{S}_{i+1}}$. The kernel of this projection is again finite dimensional and has a basis of elements of different order. As $\Sigma_{i+1}$ one takes then the union of $\widetilde{S}_{i+1}$ and the orders of the elements in this basis. In this way we obtain after a finite number of steps the desired partition $\Sigma$ of $S$.

For each $\Sigma$ in $\Im=\bigcup_{z \in Z} \Im(z)$, the elements of finite order in $F_{\Sigma}(j)$ span a dense subspace of $F_{\Sigma}(j)$ for all $j, 1 \leq j \leq m$. Hence we have in general

Corollary 3.1.12. For each flag in $F$ in $\mathfrak{\wp}$ and for each $j, 1 \leq j \leq m$, the elements of finite order in $F(j)$ span a dense subspace of $F(j)$.

For each flag $F=(F(0), \ldots, F(m))$ in $\mathfrak{F}^{(z)}$ one can also directly assign a $\Sigma(F)$ in $\Im(z)$ such that $F$ is transversal to $F_{\Sigma(F)}$. For each $1 \leq i \leq m$, put namely

$$
\Sigma(F)(i)=\{s \mid s \in S, F(i) \quad \text { contains an element of order } s\}
$$


and

$$
\Sigma(F)_{i}=\Sigma(F)(i)-\Sigma(F)(i-1) .
$$

Clearly each $F(i)$ projects bijectively onto $H_{\Sigma(F)(i)}$ and therefore $\Sigma(F)=\left\{\Sigma(F)_{i}\right\}$ belongs to $\widetilde{\subseteq}(z)$.

3.2. $\mathfrak{F}(\infty)$. In this subsection we consider some finite dimensional flag varieties contained in $\mathfrak{\Im}^{(0)}$. Let $K$ be a finite subset of S. For simplicity we assume that $K$ contains all $S_{i}$ that are finite. We denote the unitary group of $H_{K}$ by $U(K)$. It embeds naturally into $H$ by extending $u \in U(K)$ on $H_{S-K}$ by the identity. We write $\mathfrak{F}(K)$ for the subvariety of $\mathfrak{F}^{(0)}$ given by

$$
\mathfrak{\Im}(K)=\left\{u F^{(0)} \mid u \in U(K)\right\}
$$

If $K_{1} \subset K_{2}$, then we have a natural embedding of $U\left(K_{1}\right)$ into $U\left(K_{2}\right)$ and of $\mathfrak{F}\left(K_{1}\right)$ in $\mathfrak{\Im}\left(K_{2}\right)$. Now one considers a collection $\left\{K_{n} \mid n \in \mathbb{N}\right\}$ with $K_{n} \subset K_{n+1}$ for all $n$ and with $\bigcup_{n} K_{n}=S$. Then we write

$$
U(\infty)=\bigcup_{n \in \mathbb{N}} U\left(K_{n}\right) \text { and } \mathfrak{\wp}(\infty)=\bigcup_{n \in \mathbb{N}} \mathfrak{r}\left(K_{n}\right),
$$

both with the identifications mentioned above. Since the $U(\Sigma), \Sigma \in \Xi(0)$, cover $\mathfrak{F}^{(0)}$ and the "finite-size" operators are dense in $\mathfrak{I}_{2}\left(H_{j}, H_{i}\right)$, we get

Lemma 3.2.1. The space $\mathfrak{F}(\infty)$ lies dense in $\mathfrak{F}^{(0)}$.

Hence, by restricting holomorphic functions on $\mathfrak{r}^{(0)}$ to $\mathfrak{F}(\infty)$ we get

Corollary 3.2.2. The only holomorphic functions from $\mathfrak{\Im}^{(0)}$ to $\mathbb{C}$ are the constants.

3.3. Another description of $\mathfrak{F}^{(0)}$. For each $\Sigma$ in $\subseteq(z)$, there are numerous $\underline{\sigma} \in W$ such that $F_{\Sigma}=\underline{\sigma} F^{(0)}$. We start by describing a special choice that is useful at the description of $\widetilde{\Im}^{(0)}$ as the homogeneous space of another group. Recall that we had for $i \geq 3$ the notation

$$
S_{i}=\left\{s_{i}(k) \mid 1 \leq k<m_{i}+1\right\}
$$

In order to make this notation uniform we write

$$
s_{1}(k)=k-1 \text {, for } 1 \leq k<m_{1}+1 \text {, and } s_{2}(k)=-k \text {, for } 1 \leq k<m_{2}+1 \text {. }
$$

Now we construct a bijection $\sigma_{i}: S_{i} \rightarrow \Sigma_{i}$, as follows: since $S_{i}$ and $\Sigma_{i}$ are commensurable, there is a $N \geq 0$ such that

$$
\left\{s_{i}(k) \mid k>N\right\} \subset \Sigma_{i} \cap S_{i} .
$$

Consider the finite set $\Sigma_{i}-\left\{s_{i}(k) \mid k>N\right\}$. We may assume that it is ordered increasingly if $i=1$ and decreasingly if $i \geq 2$. This means $\Sigma_{i}-\left\{s_{i}(k) \mid k>N\right\}=$ $\left\{t_{k} \mid k<N+\ell_{i}\right\}$ for some $\ell_{i}$ in $\mathbb{Z}$ and

$$
t_{1}<t_{2}<\ldots<t_{N+\ell_{i}}, \quad \text { or } t_{1}>t_{2}>\ldots>t_{N+\ell_{i}} .
$$


Now we can define $\sigma_{i}: S_{i} \rightarrow \Sigma_{i}$ by putting

$$
\begin{array}{ll}
\sigma_{i}\left(s_{i}(k)\right)=t_{k} & \text { for } 1 \leq k \leq N+\ell_{i}, \\
\sigma_{i}\left(s_{i}(k)\right)=s_{i}\left(k-\ell_{i}\right) & \text { for } k>N+\ell_{i} .
\end{array}
$$

The index of $p_{i} \circ \underline{\sigma}_{i}$ is equal to that of $\underline{\tau}_{i}: H_{i} \rightarrow H_{i}$, where $\tau_{i}: S_{i} \rightarrow S_{i}$ is defined by

$$
\begin{array}{ll}
\tau_{i}\left(s_{i}(k)\right)=s_{i}(N+1) & \text { for all } 1 \leq k \leq N+\ell_{i} \\
\tau_{i}\left(s_{i}(k)\right)=s_{i}\left(k-\ell_{i}\right) & \text { for all } k>N+\ell_{i} .
\end{array}
$$

Clearly we have ind $\left(\underline{\tau}_{i}\right)=N+\ell_{i}-N=\ell_{i}$. In other words, the number $\ell_{i}$ is equal to $z_{i}$. The $\left\{\sigma_{i} \mid 1 \leq i \leq m\right\}$ compose to a bijection $\sigma: S \rightarrow S$ such that $\underline{\sigma} F^{(0)}=F_{\Sigma}$. We will introduce a special term for this type of permutations.

Definition 3.3.1. Let $\Sigma$ be a partition of $S$ in $\subseteq(z)$. A permutation $\sigma$ of $S$ such that $\sigma\left(S_{i}\right)=\Sigma_{i}$ is called admissible of level $N$ if the following properties hold

(i) For each $i$ and for all $k>N+z_{i}, \sigma\left(s_{i}(k)\right)=s_{i}\left(k-z_{i}\right)$.

(ii) For all $k_{1}$ and $k_{2} \leq N+z_{i}$ and all $1 \leq i \leq m, s_{i}\left(k_{1}\right) \geq s_{i}\left(k_{2}\right) \Rightarrow \sigma\left(s_{i}\left(k_{1}\right)\right) \geq$ $\sigma\left(s_{i}\left(k_{2}\right)\right)$.

Now we can introduce another group that acts transitively on $\mathfrak{F}^{(0)}$. Its advantage is that it enables you to construct in a simple way holomorphic line bundles over $\mathfrak{F}^{(0)}$.

Let $\Sigma$ be in $\widetilde{\subseteq}(0)$ and let $\sigma$ be an admissible permutation of $S$ such that $\sigma\left(H_{i}\right)=$ $\Sigma_{i}$. From the definition of admissibility we know that $\underline{\sigma}$ decomposes in operators $\left(\underline{\sigma}_{i j}\right)$ with the properties

(i) For each $1 \leq i \leq m, \underline{\sigma}_{i i}=I d_{H_{i}}+$ a "finite-size" operator.

(ii) For all $i$ and $j, i \neq j, \underline{\sigma}_{i j}$ is a "finite-size" operator.

Since every flag $F$ in $\mathfrak{\Im}^{(0)}$ is transversal to some $F_{\Sigma}$, with $\Sigma$ in $\widetilde{\varsigma}(0)$, we may conclude that each $F$ in $\mathfrak{F}^{(0)}$ is equal to $g . F^{(0)}$ with $g \in G l_{\text {res }}(H)$ of the form

(a) For each $i, 1 \leq i \leq m, g_{i i}=I d_{H_{i}}+$ a "finite-size" operator.

(b) For all $i$ and $j, i<j, g_{i j}$ is a "finite-size" operator.

(c) For all $i$ and $j, j<i, g_{i j}$ belongs to $\mathfrak{I}_{2}\left(H_{j}, H_{i}\right)$.

For the operators $g_{i i}$ from (a) there always exists a determinant. As is well-known, see [7], one can take the determinant of any operator of the form "identity + a nuclear operator". Therefore we introduce

Notation 3.3.2. If $K_{1}$ and $K_{2}$ are complex Hilbert spaces, then we denote the space of nuclear operators from $K_{1}$ to $K_{2}$ by $\Re\left(K_{1}, K_{2}\right)$ and the tracenorm on it by $\|\cdot\|_{t r}$. If $K_{1}$ equals $K_{2}$, then we simply write $\mathfrak{i}\left(K_{1}\right)$ instead of $\mathfrak{U}\left(K_{1}, K_{2}\right)$.

The desire to work in a Banach context and the fact that we like to be able to take determinants of operators, make you consider the collection $B_{2}(H)$ of operators in 
$B_{\text {res }}(H)$ given by

$$
B_{2}(H)=\left\{g \mid g \in B_{\text {res }}(H), \begin{array}{ll}
g_{i i}-I d_{H_{i}} \in \mathfrak{N}\left(H_{i}\right) & \text { for all } i \\
g_{i j} \in \mathfrak{I}_{2}\left(H_{j}, H_{i}\right) & \text { for } j \neq i
\end{array}\right\} .
$$

On $B_{2}(H)$ we put a different topology than the one induced by $B_{\text {res }}(H)$. For, let (5) be the subspace of $B_{\text {res }}(H)$ defined by

$$
\mathfrak{S}=\left\{b \mid b \in B_{\text {res }}(H), \quad \begin{array}{ll}
b_{i i} \in \mathfrak{N}\left(H_{i}\right) & \text { for all } i \\
b_{i j} \in \mathfrak{I}_{2}\left(H_{j}, H_{i}\right) & \text { for all } j \neq i
\end{array}\right\}
$$

Then 5 becomes a Banach space if we equip it with the norm $\|\cdot\|_{E}$ given by

$$
\|b\|_{E}=\sum_{j \neq i}\left\|b_{i j}\right\|_{H S}+\sum_{i}\left\|b_{i i}\right\|_{t r} .
$$

The map $g \mapsto g-I d$ permits you to transfer this Banach space structure from ${ }_{5}$ to $B_{2}(H)$. Note that the subgroup $U_{-}$of $G l_{\text {res }}(H)$ defined by

$$
U_{-}=\left\{g \mid g \in G l_{\mathrm{res}}(H), g_{i i}=I d_{H_{i}} \text { for all } i, g_{i j}=0 \text { for all } i<j\right\}
$$

acts on $B_{2}(H)$ by left multiplication and its "adjoint" $U_{+}$, defined by

$$
U_{+}=\left\{u^{*} \mid u \in U_{-}\right\},
$$

acts by right translation on $B_{2}(H)$. Both actions are analytic w.r.t. the 5 -structure on $B_{2}(H)$. Let $b=\left(b_{i j}\right)$ belong to $B_{2}(H)$. Since each $b_{i i}$ has the form $I d_{H_{i}}+$

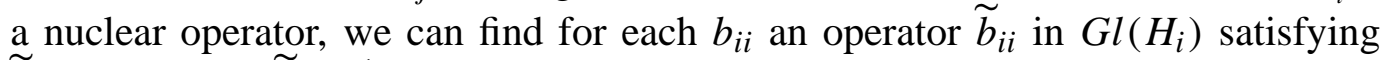
$\widetilde{b}_{i i}-I d_{H_{i}}$ and $\left(\widetilde{b}_{i i}\right)^{-1}-I d_{H_{i}} \in \mathfrak{N}\left(H_{i}\right)$. Then one verifies directly that if $u=\left(u_{i j}\right)$ in $U_{-}$and $v=\left(v_{i j}\right)$ in $U_{+}$are defined by

$$
\begin{gathered}
u_{i i}=v_{i i}=I d_{H_{i}}, u_{i j}=-b_{i j}\left(\tilde{b}_{j j}\right)^{-1} \quad \text { if } i>j, u_{i j}=0 \quad \text { if } j>i . \\
v_{i j}=-\left(\tilde{b}_{i i}\right)^{-1} b_{i j} \quad \text { if } i<j \text { and } \quad v_{i j}=0 \quad \text { if } i>j .
\end{gathered}
$$

then the operator $u . b . v-I d$ belongs to $\mathfrak{N}(H)$. This result we put in a

Lemma 3.3.3. Every $b \in B_{2}(H)$ can be decomposed as $b=u b_{1} v$, where $u$ belongs to $U_{-}, b_{1}$ belongs to $I d+\mathfrak{I}(H)$ and $v$ belongs to $U_{+}$.

If we take into account that for each $i$, the operator $\widetilde{b}_{i i}$ can be chosen of the form $b_{i i}+f_{i i}$, with $f_{i i}$ a finite dimensional operator, then for each $c$ in $B_{2}(H)$ sufficiently close to $b$, we can take $\widetilde{c}_{i i}=c_{i i}+f_{i i}$. By using this, one shows easily that the map det $: B_{2}(H) \rightarrow \mathbb{C}$, defined by

$\operatorname{det}(b)=\operatorname{det}\left(b_{1}\right), \quad$ where $b=u b_{1} v$, with $u \in U_{-}, b_{1} \in I d+\mathfrak{A}(H)$ and $v \in U_{+}$, is analytic on $B_{2}(H)$. Now we are interested in the elements of $B_{2}(H)$ that belong to $G l_{\text {res }}(H)$, i.e. in

$$
G_{2}=\left\{g \mid g \in B_{2}(H) \cap G l_{\text {res }}(H)\right\} .
$$


One easily verifies that $G_{2}$ is a group and, since it is known that an element $g$ of $I d+\mathfrak{i}(H)$ is invertible if and only if $\operatorname{det}(g) \neq 0$, we may conclude that $G_{2}$ is an open part on $B_{2}(H)$ and thus inherits a Banach structure. It is a straightforward verification that $G_{2}$ is a Banach Lie group that acts analytically on $\mathfrak{F}^{(0)}$. As we have seen above this action is transitive and, if we denote the stabilizer of $F^{(0)}$ in $G_{2}$ by

$$
\mathfrak{I}=\left\{t=\left(\begin{array}{ccc}
t_{11} & & t_{1 m} \\
0 & \ddots & \\
\vdots & \ddots & \\
0 & 0 & t_{m m}
\end{array}\right) \mid \begin{array}{l}
t_{i i} \in\left\{I d+\mathfrak{A}\left(H_{i}\right)\right\} \cap G L\left(H_{i}\right), \\
t_{i j} \in \mathfrak{I}_{2}\left(H_{j}, H_{i}\right) \text { for } j>i
\end{array}\right\},
$$

then we can identify $\mathfrak{F}^{(0)}$ with the homogeneous space $G_{2} / \mathfrak{I}$.

Remark 3.3.4. One can give the same type of description for the other components $\mathfrak{\Im}^{(z)}$, by taking some $\Sigma \in \Im_{(z)}$ and by introducing the group $G_{2}$ as the operators that decompose w.r.t. $H=H_{\Sigma_{1}} \oplus \cdots \oplus H_{\Sigma_{m}}$ in the above way.

3.4. The line bundles and the central extension. For every $\underline{k}=\left(k_{1}, \ldots, k_{m}\right)$ in $\mathbb{Z}^{m}$, we can define a holomorphic character $\chi_{\underline{k}}$ of the group $\mathfrak{I}$ by

$$
\chi_{\underline{k}}(t)=\operatorname{det}\left(t_{11}\right)^{k_{1}} \ldots \operatorname{det}\left(t_{m m}\right)^{k_{m}} .
$$

To this character is associated a line bundle $L(\underline{k})$ over $\mathfrak{F}^{(0)}=G_{2} / \mathfrak{I}$. It is defined as follows: consider on the space $G_{2} \times \mathbb{C}$ the equivalence relation

$$
\left(g_{1}, \lambda_{1}\right) \sim\left(g_{2}, \lambda_{1}\right) \Leftrightarrow g_{1}=g_{2} \circ t, \quad \text { with } t \in \mathfrak{I} \text { and } \lambda_{2}=\lambda_{1} \chi_{\underline{k}}(t) .
$$

The space $G_{2} \times \mathbb{C}$ modulo this equivalence relation is $L(\underline{k})$. For each $g \in G_{2}$ and each $\lambda$ in $\mathbb{C}$, we denote the equivalence class to which the pair $(g, \lambda)$ belongs by $[g, \lambda]$. There is a natural projection $\pi: L(\underline{k}) \rightarrow \mathfrak{F}^{(0)}$ given by

$$
\pi([g, \lambda])=g . F^{(0)} \text {. }
$$

It is a direct verification to show that $L(\underline{k})$ is a Hilbert manifold based on the Hilbert space

$$
\bigoplus_{j<i} \mathfrak{I}_{2}\left(H_{j}, H_{i}\right) \oplus \mathbb{C}
$$

and that $L(\underline{k})$ is a holomorphic line bundle over $\mathfrak{r}^{(0)}$.

Remark 3.4.1. The line bundles $L(\underline{k})$ are natural infinite dimensional generalizations of the holomorphic line bundles over the space of flags in $\mathbb{C}^{n}$ of a fixed length and of a fixed size. In the case of the Grassmann manifold from example 2.1.3, the bundles $L((-1,0))$ and $L((1,0))$ correspond respectively to the determinant bundle Det and its dual Det* that play an important role in the connection with integrable systems. 
We have a natural action of the group $G_{2}$ on the space $L(\underline{k})$, namely by

$$
g_{1} \cdot[g, \lambda]=\left[g_{1} g, \lambda\right]
$$

This is a lifting of the natural action of $G_{2}$ on $\mathfrak{F}^{(0)}$ to one on $L(\underline{k})$. However, the natural action of $G l_{\text {res }}^{(0)}(H)$ on $\mathfrak{F}^{(0)}$ can, in general, not be lifted to one on $L(\underline{k})$. This has been shown in [20] for the Grassmann manifold from example 2.1.3 and the bundle $L((-1,0))$. If one wants to lift the action to $L(\underline{k})$ it is necessary to pass to an extension of $G l_{\mathrm{res}}^{(0)}(H)$. Note that for each $g$ in $G l_{\mathrm{res}}^{(0)}(H)$ there exists a $p$ in $P$ such that $g p^{-1} \in G_{2}$. Hence if we define the group

$$
G=\left\{(g, p) \mid g \in G l_{\text {res }}^{(0)}(H), p \in P, g p^{-1} \in G_{2}\right\} .
$$

Then $G$ is an extension of $G l_{\text {res }}^{(0)}(H)$ that acts on $L(\underline{k})$ by

$$
(g, p) \cdot\left[g_{1}, \lambda_{1}\right]=\left[g g_{1} p^{-1}, \lambda_{1}\right] .
$$

This action clearly lifts the action of $G$, via the projection $\pi_{1}: G \rightarrow G l_{\text {res }}^{(0)}(H)$. We assume that $\mathfrak{I}$ is embedded into $G$ by means of $t \mapsto(I d, t)$. In $\mathfrak{I}$ we have a normal subgroup

$$
\mathfrak{I}_{1}=\left\{(I d, t) \mid t \in \mathfrak{I}, \chi_{\underline{k}}(t)=1\right\} .
$$

The group $\mathfrak{I}_{1}$ acts trivially on the space $L(\underline{k})$ so we have in fact an action of $\widetilde{G}=$ $G / \mathfrak{I}_{1}$ on $L(\underline{k})$. The group $\widetilde{G}$ is a central extension of $G l_{\text {res }}^{(0)}(H)$ with the group $\mathfrak{I} / \mathfrak{I}_{1}$. In general this extension is non-trivial.

Remark 3.4.2. The central extension $\widetilde{G}$ is determined by a 2-cocycle on $G l_{\text {res }}^{(0)}(H)$. If one computes for $m=2, m_{1}=m_{2}=\infty$ and $\underline{k}=(1,0)$, the corresponding Lie algebra 2-cocycle, then this gives you on $\overline{g l}_{\infty} \cap\left[B_{\text {res }}(H)\right]$, up to an irrelevant factor, the one defining $A_{\infty}$.

3.5. The holomorphic sections of $\boldsymbol{L}(\underline{\boldsymbol{k}})$. Let $\mathfrak{R}(\underline{k})$ denote the space of holomorphic sections of $L(\underline{k})$. The space $\mathfrak{Q}(\underline{k})$ is given the topology of uniform convergence on compact subsets of $\mathfrak{F}^{(0)}$. It becomes then a complete locally convex space, see [13]. Let $\underline{f}: \mathfrak{F}^{(0)} \rightarrow L(\underline{k})$ belong to $\mathfrak{R}(\underline{k})$ then it can be written as

$$
\underline{f}\left(g \cdot F^{(0)}\right)=[g, f(g)], \quad \text { for all } g \in G_{2},
$$

where $f: G_{2} \rightarrow \mathbb{C}$ is a holomorphic function satisfying

$$
f(g t)=f(g) \chi_{\underline{k}}(t)^{-1} \quad \text { for all } g \in G_{2} \text { and all } t \in \mathfrak{I} .
$$

Thus we can identify $\mathfrak{L}(\underline{k})$ with the space of holomorphic functions on $G_{2}$ that satisfy this condition. Since each $(g, p)$ in $G$ acts as an analytic diffeomorphism on as well $\mathfrak{r}^{(0)}$ as $L(\underline{k})$, we get a natural action of $G$ on $\mathfrak{L}(\underline{k})$ that corresponds on the functions on $G_{2}$ to

$$
(g, p)(f)\left(g_{1}\right)=f\left(g^{-1} g_{1} p\right), \quad \text { with } g_{1} \in G_{2} \text { and }(g, p) \in G .
$$


The space $\mathfrak{L}(\underline{k})$ can be zero, as we know from the finite dimensional case. We are interested in knowing when this is the case. Assume first that $\underline{k} \in \mathbb{Z}^{m}$ satisfies

$$
k_{1} \leq k_{2} \ldots \leq k_{m}=0 .
$$

Then we will construct concrete non-zero elements of $\mathfrak{L}(\underline{k})$. If $\Sigma=\left\{\Sigma_{j}\right\}$ belongs to $\subseteq(0)$, then we write

$$
\Sigma(i)=\bigcup_{j \leq i} \Sigma_{j} \quad \text { and } \quad \Im_{i}=\{\Sigma(i) \mid \Sigma \in \Xi(0)\} .
$$

Let $\sigma=\sigma_{1} \oplus \ldots \oplus \sigma_{m}$ be an admissible permutation of $S$ corresponding to $\Sigma$. Consider for $g \in G_{2}$ the operator $\left(\underline{\sigma}_{1} \oplus \ldots \oplus \underline{\sigma}_{i}\right)^{-1} \circ p_{\Sigma(i)} \circ g \mid \bigoplus_{j \leq i} H_{j}$ from $\bigoplus_{j \leq i} H_{j}$ to itself. It decomposes as

$$
\left(\begin{array}{ccc}
h_{11} & \ldots & h_{1 i} \\
\vdots & \ddots & \vdots \\
h_{i 1} & \ldots & h_{i i}
\end{array}\right) \text {, with } h_{j j}-I d_{H_{j}} \in \mathfrak{A}\left(H_{j}\right), h_{i j} \in \mathfrak{I}_{2}\left(H_{j}, H_{i}\right) \text { for all } j \neq i \text {. }
$$

In particular we can take the determinant of this operator. The function $f_{\Sigma(i)}$ : $G_{2} \rightarrow \mathbb{C}$ defined by

$$
f_{\Sigma(i)}(g)=\operatorname{det}\left(\left(\underline{\sigma}_{1} \oplus \ldots \oplus \underline{\sigma}_{i}\right)^{-1} \circ p_{\Sigma(i)} \circ g \mid \bigoplus_{j \leq i} H_{j}\right)
$$

satisfies for each $t=\left(t_{i j}\right)$ in $\mathfrak{I}$ the condition

$$
f_{\Sigma(i)}(g t)=f_{\Sigma(i)}(g) \operatorname{det}\left(t_{11}\right) \ldots \operatorname{det}\left(t_{i i}\right)
$$

In other words $f_{\Sigma(i)}$ belongs to $\mathfrak{Q}((-1, \ldots,-1,0, \ldots))$. Let $P_{j}, 1 \leq j \leq m-1$, be a homogeneous polynomial in the $\left\{f_{\Sigma(j)} \mid \Sigma(j) \in \widetilde{\Xi}_{j}\right\}$ of degree $k_{j+1}-k_{j}$ then it will be clear that $\prod_{j=1}^{m-1} P_{j}$ is a non-zero element of $\mathfrak{L}(\underline{k})$. Next we consider the case that $\underline{k} \in \mathbb{Z}^{m}$ satisfies

$$
k_{1} \leq \ldots \leq k_{m}
$$

We take the $\left\{P_{i} \mid i<m\right\}$ as above and we choose for $P_{m}$ the function

$$
g \mapsto \operatorname{det}(g)^{-k_{m}} .
$$

the function $\prod_{i=1}^{m} P_{i}$ defines then a non-zero element of $\mathfrak{L}(\underline{k})$. By restricting sections of $L(\underline{k})$ to the dense collection $\mathfrak{F}(\infty)$ of "finite flags" in $\mathfrak{F}^{(0)}$ and exploiting the finite dimensional results one obtains

\section{Theorem 3.5.1.}

(a) The space $\mathfrak{Q}(\underline{k})$ is non-zero if and only if $k_{1} \leq \ldots \leq k_{m}$ 
(b) If $\mathfrak{Q}(\underline{k})$ is non-zero, then the functions $\prod_{i=1}^{m} P_{i}\left(\left\{f_{\Sigma(i)} \mid \Sigma(i) \in \widetilde{\Xi}_{i}\right\}\right)$ span a dense subspace of $\mathfrak{R}(\underline{k})$.

Next we consider the representation of $\widetilde{G}$ on $\mathfrak{L}(\underline{k})$. Then each closed non-zero subspace of $\mathfrak{L}(\underline{k})$ can be shown to contain a non-zero vector that is $U(K)$-finite for some finite $K$ in $S$. By analyzing the restriction map at increasing $K$, by applying the action of $U(\infty)$ and by using the finite dimensional results one obtains

Theorem 3.5.2. The action of $G_{2}$ (and hence of $\widetilde{G}$ on $\mathfrak{Q}(\underline{k})$ ) is irreducible.

After this description of the geometry of this infinite dimensional flag variety $\mathfrak{F}$ and this collection of line bundles over $\mathfrak{F}^{(0)}$, we will give a link with some integrable systems and we start by giving a description of the system of non-linear equations we are interested in.

\section{Appl ications to int egrable syst ems}

4.1. The multicomponent KP-hierarchy. The formal set-up of this system of equations is as follows: consider a complex commutative differential algebra $R$ with a collection of commuting derivations $\left\{\partial_{i \alpha} \mid i \geq 1,1 \leq \alpha \leq r\right\}$ of $R$. Let $\partial$ be the derivation $\sum_{\alpha=1}^{r} \partial_{1 \alpha}$. The equations of the hierarchy can be formulated conveniently in terms of relations for certain elements from the ring $g l_{r}(R)\left(\left(\partial, \partial^{-1}\right)\right)$ of pseudodifferential operators in $\partial$ with coefficients from $g l_{r}(R)$. We extend the $\partial_{i \alpha}$ to derivations of $g l_{r}(R)\left(\left(\partial, \partial^{-1}\right)\right)$ by letting it act coefficientwise on elements of $g l_{r}(R)$ and on an element $\sum_{j \leq N} p_{j} \partial^{j}$ of $g l_{r}(R)\left(\left(\partial, \partial^{-1}\right)\right)$ by

$$
\partial_{i \alpha}\left(\sum_{j \leq N} p_{j} \partial^{j}\right)=\sum_{j \leq N} \partial_{i \alpha}\left(p_{j}\right) \partial^{j}
$$

In the ring $g l_{r}(R)\left(\left(\partial, \partial^{-1}\right)\right)$ we denote the differential operator part $\sum_{j \geq 0} p_{j} \partial^{j}$ of $P=\sum_{j} p_{j} \partial^{j}$ by $P_{+}$and we write $P_{-}$for $P-P_{+}$.

Let $E_{\alpha}, 1 \leq \alpha \leq r$, be the diagonalmatrix in $g l_{r}(\mathbb{C})$ with $(\alpha, \alpha)$-entry equal to 1 and the other entries equal to zero. In the ring $g l_{r}(R)\left(\left(\partial, \partial^{-1}\right)\right)$ we consider elements of the form

$$
L=\partial+\sum_{j>0} \ell_{j} \partial^{-j} \quad \text { and } \quad U_{\alpha}=E_{\alpha}+\sum_{j>0} u_{\alpha j} \partial^{-j}
$$

Examples of this type of operators can be obtained as follows: take the trivial example $L=I d \partial=\partial$ and $U_{\alpha}=E_{\alpha}$ and choose some $K=I d+\sum_{j>0} k_{j} \partial^{-j}$ in 
$g l_{r}(R)\left(\left(\partial, \partial^{-1}\right)\right)$. Such a $K$ is invertible and

$$
L=K \partial K^{-1} \quad \text { and } \quad U_{\alpha}=K E_{\alpha} K^{-1}
$$

have the form (4). Following [23], the equations of the multicomponent KPhierarchy are

$$
\begin{aligned}
{\left[L, U_{\alpha}\right] } & =\left[U_{\alpha}, U_{\beta}\right]=0 \\
U_{\alpha} U_{\beta} & =\delta_{\alpha \beta} U_{\beta} \\
\delta_{i \alpha}(L) & =\left[\left(L^{i} U_{\alpha}\right)_{+}, L\right]:=\left[B_{i \alpha}, L\right] \\
\delta_{i \alpha}\left(U_{\beta}\right) & =\left[B_{i \alpha}, U_{\beta}\right] .
\end{aligned}
$$

The equations (6) and (7) are satisfied for all elements $L$ and $\left\{U_{\alpha}\right\}$ of the form (5). The equations (8) and (9) boil down to non-linear differential equations for the $\left\{u_{\alpha j}\right\}$ and $\left\{\ell_{j}\right\}$. Since all the solutions of the multicomponent KP-hierarchy that we will construct are of the form (5), we merely have to focuss on (8) and (9). These last equations can be seen as compatibility conditions for a linear system. This requires the introduction of a $g l_{r}(R)\left(\left(\partial, \partial^{-1}\right)\right)$-module. Let $M$ consist of the formal products

$$
\left\{\sum_{j=-\infty}^{N} \beta_{j} \lambda^{j}\right\} \exp \left(\sum_{\substack{i \geq 1 \\ 1 \leq \alpha \leq r}} t_{i \alpha} E_{\alpha} \lambda^{i}\right)=\left\{\sum_{j=-\infty}^{N} \beta_{j} \lambda^{j}\right\} g(\lambda),
$$

with $\beta_{j} \in g l_{r}(R)$. For $\beta \in g l_{r}(R)$, the action of $\beta$ is defined by

$$
\beta\left\{\sum_{j=-\infty}^{M} \beta_{j} \lambda^{j}\right\} g(\lambda)=\left\{\sum_{j=-\infty}^{M} \beta \beta_{j} \lambda^{j}\right\} g(\lambda) .
$$

The action of $\partial_{i \alpha}$ on $M$ is defined such that it corresponds to "differentiating" this formal product w.r.t. the variable $t_{i \alpha}$, i.e.

$$
\partial_{i \alpha}\left(\left\{\sum_{j} b_{j} \lambda^{j}\right\} g(\lambda)\right)=\left\{\sum_{j} \partial_{i \alpha}\left(b_{j}\right) \lambda^{j}+\sum_{j} b_{j} E_{\alpha} \lambda^{i+j}\right\} g(\lambda) .
$$

In particular we see that the action of $\partial$ on $M$

$$
\partial\left\{\sum b_{j} \lambda^{j}\right\} g(\lambda)=\sum\left\{\partial\left(b_{j}\right) \lambda^{j}+\sum b_{j} \lambda^{j+1}\right\} g(\lambda)
$$

is invertible with the inverse $\partial^{-1}$ given by

$$
\partial^{-1}\left\{\sum b_{j} \lambda^{j}\right\} g(\lambda)=\left\{\sum_{i=0}^{\infty} \sum_{j}(-1)^{i} \partial^{i}\left(b_{j}\right) \lambda^{j-i-1}\right\} g(\lambda) .
$$

These actions compose to a $g l_{r}(R)\left(\left(\partial, \partial^{-1}\right)\right)$-module structure on $M$. In fact, $M$ is a free $g l_{r}(R)\left(\left(\partial, \partial^{-1}\right)\right)$-module with generator $g(\lambda)$, for, if $P=\Sigma P_{j} \partial^{j} \in$ 
$g l_{r}(R)\left(\left(\partial, \partial^{-1}\right)\right)$, then

$$
P \cdot g(\lambda)=\left\{\sum P_{j} \lambda^{j}\right\} g(\lambda)
$$

Let $\Delta$ be the subgroup of $g l_{r}\left(\mathbb{C}\left(\lambda, \lambda^{-1}\right)\right)$ given by

$$
\Delta=\left\{\delta \mid \delta=\operatorname{diag}\left(\lambda^{k_{1}}, \ldots, \lambda^{k_{r}}\right) \text { with }\left(k_{1}, \ldots, k_{r}\right) \in \mathbb{Z}^{r}\right\} .
$$

Take any $\delta=\Sigma d_{j} \lambda^{j}, d_{j} \in g l_{r}(\mathbb{C})$, in $\Delta$. To $\delta$ corresponds the element $\underline{\delta}=\Sigma d_{j} \partial^{j}$ in $g l_{r}(R)\left(\left(\partial, \partial^{-1}\right)\right)$. Then we have the notion

Definition 4.1.1. A function of type $\delta$ is an element of $\psi$ of $M$ that has the form

$$
\psi=\left\{\left(I d+\sum_{j<0} \psi_{j} \lambda^{j}\right)\left(\sum_{k} d_{k} \lambda^{k}\right)\right\} g(\lambda) .
$$

To any function $\psi$ of type $\delta$ we associate the operator $K_{\psi}$ in $g l_{r}(R)\left(\left(\partial, \partial^{-1}\right)\right)$ given by

$$
K_{\psi}=I d+\sum_{j} \psi_{j} \partial^{j}
$$

Next we assume that we have been given operators $L$ and $\left\{U_{\alpha}\right\}$ of the form (5). Then we introduce the following notion:

Definition 4.1.2. A wavefunction of type $\delta$ for $L$ and the $\left\{U_{\alpha}\right\}$ is a function $\psi$ of type $\delta$ satisfying

(a) $L(\psi)=\lambda \psi$

(b) $U_{\alpha} \psi=\psi E_{\alpha}$

(c) $\partial_{i \alpha}(\psi)=P_{i \alpha} \cdot \psi$ with $P_{i \alpha} \in g l_{r}(R)[\partial]$.

The first two properties translate respectively into

$$
L=K_{\psi} \partial K_{\psi}^{-1} \quad \text { and } \quad U_{\alpha}=K_{\psi} E_{\alpha} K_{\psi}^{-1}
$$

Hence $L$ and the $\left\{U_{\alpha}\right\}$ are completely determined by $\psi$. One computes directly that (c) implies $P_{i \alpha}=\left(L^{i} U_{\alpha}\right)_{+}$and by applying the operators $\partial_{i \alpha}$ to the equations (a) and (b) and by substituting (c) one shows

Theorem 4.1.3. If $\psi$ is a wavefunction of type $\delta$, then the operators $K_{\psi} \partial K_{\psi}^{-1}$ and $\left\{K_{\psi} E_{\alpha} K_{\psi}^{-1}\right\}$ satisfy the equations of the multicomponent KP-hierarchy.

Now we are ready to describe the geometric setting from which we can construct wavefunctions of type $\delta$. 
4.2. The commuting flows. Let $H$ be the Hilbert space $L^{2}\left(S^{1}, \mathbb{C}^{r}\right)$ with the usual norm. Let $\left\{f_{\ell} \mid 1 \leq \ell \leq r\right\}$ be the standard basis of $\mathbb{C}^{r}$. Then the elements of $H$ are written as

$$
\sum_{i \in \mathbb{Z}} \sum_{k=1} \alpha_{i k} f_{k} \lambda^{i}, \quad \text { with } \alpha_{i k} \in \mathbb{C} .
$$

The space $H$ is decomposed as $H=H_{1} \oplus H_{2}$ with

$$
H_{1}=\left\{\sum_{i \geq 0} h_{i} \lambda^{i} \mid h_{i} \in \mathbb{C}^{r}\right\} \quad \text { and } H_{2}=H_{1}^{\perp} .
$$

The elements $\left\{f_{k} \lambda^{i} \mid 1 \leq k \leq r, i \geq 0\right\}$ are an orthonormal basis of $H_{1}$ and the $\left\{f_{k} \lambda^{i} \mid i<0,1 \leq k \leq r\right\}$ one of $\bar{H}_{2}$. To get a numbering like in the foregoing section, one defines $e_{k+i r-1}=f_{k} \lambda^{i}$. In the present context it is also convenient to see the matrix $[g]$ of an operator $g$ in $B_{\text {res }}(H)$ as an $\mathbb{Z} \times \mathbb{Z}$-matrix with entries in $g l_{r}(\mathbb{C})$, i.e.

$$
[g]=\left(\begin{array}{cccc}
\ddots & & & \\
& G_{k+1 \ell+1} & G_{k+1 \ell} & \\
& G_{k \ell+1} & G_{k \ell} & \\
& & & \ddots
\end{array}\right), \quad \text { with } G_{s t} \in g l_{r}(\mathbb{C}) .
$$

An important operator in $B_{\text {res }}(H)$ is the multiplication $\Lambda$ on each factor with $\lambda$. It has the matrix $[\Lambda]$ with $\Lambda_{i i-1}=I d$ and $\Lambda_{i j}=0$, if $j \neq i-1$. One verifies directly that the centralizer $Z(\Lambda)$ of $\Lambda$ in $B_{\text {res }}(H)$ consists of all $g$ in $B_{\text {res }}(H)$ such that the matrix of $g$ looks like

$$
[g]=\left(\begin{array}{ccccc}
\ddots & \ddots & & & \\
\ddots & G_{00} & G_{10} & & \\
& G_{-10} & G_{00} & G_{10} & \ddots \\
& & G_{-10} & G_{00} & \ddots \\
& & & \ddots &
\end{array}\right) .
$$

Clearly, multiplying with an $A$ from $g l_{r}(\mathbb{C})$, defines an element of $B_{\text {res }}(H)$. Let $\mathfrak{h}$ be the diagonal matrices in $g l_{r}(\mathbb{C})$. It is obvious then that

$$
\left\{\sum_{i \in \mathbb{Z}} H_{i} \Lambda^{i} \in B_{\text {res }}(H), H_{i} \in \mathfrak{h} \quad \text { for all } i\right\}
$$

is a maximal commutative subalgebra of $B_{\text {res }}(H)$. The group of commuting flows that we will consider is contained in this algebra and takes care of essentially all independent directions. To be more precise, let $U$ be a connected neighborhood of $S^{1}$ in $\mathbb{C}$ and let $\Gamma(U)$ be the space of all analytic maps $\gamma: U \rightarrow \mathfrak{h}$ such that $\operatorname{det}(\gamma(u)) \neq 0$ for all $u \in U$. In a natural way $\Gamma(U)$ is a group. If $U_{1} \supset U_{2}$ then we 
get an embedding of $\Gamma\left(U_{1}\right)$ into $\Gamma\left(U_{2}\right)$ by restricting functions to $U_{2}$. We write $\Gamma$ for the direct limit of the $\{\Gamma(U)\}$. Each $\gamma \in \Gamma$ has a Fourier series

$$
\gamma=\sum_{i \in \mathbb{Z}} \gamma_{i} \lambda^{i}, \gamma_{i} \in \mathfrak{h}
$$

The multiplication with $\gamma$, defines the element $\sum_{i \in \mathbb{Z}} \gamma_{i} \Lambda^{i}$ in $B_{\text {res }}(H)$. At the consideration of the flows from $\Gamma$ on $\mathfrak{\Im}$ we make use of a decomposition of the elements of $\Gamma$. In $\Gamma$ we consider namely the following subgroups

$$
\begin{aligned}
& \Gamma_{+}=\left\{\gamma \mid \gamma \in \Gamma, \gamma=\exp \left(\sum_{\substack{i>0 \\
1 \leq \alpha \leq r}} t_{i \alpha} E_{\alpha} \lambda^{i}\right)\right\}, \\
& \Gamma_{-}=\left\{\gamma \mid \gamma=\sum_{j \leq 0} \gamma_{j} \lambda^{j} \in \Gamma\right\}
\end{aligned}
$$

and $\Delta=\left\{\delta \mid \delta=\operatorname{diag}\left(\lambda^{k_{1}}, \ldots, \lambda^{k_{r}}\right)\right.$ with $k_{i} \in \mathbb{Z}$ for all $\left.i\right\}$. Then there holds

Lemma 4.2.1. The group $\Gamma$ decomposes as $\Gamma=\Gamma_{+} \Delta \Gamma_{-}$.

This lemma is a direct consequence of the decomposition of holomorphic line bundles over $\mathbb{P}^{1}(\mathbb{C})$, see [6].

4.3. The solutions. Since $m=2$, all flags in $\widetilde{F}$ correspond to subspaces $W$ of $H$. For each $W$ in $\mathfrak{F}$, consider

$$
\Delta_{W}=\left\{\delta \mid \delta \in \Delta, \text { there is a } \gamma \in \Gamma_{+} \text {such that } \gamma^{-1} \delta^{-1} W \text { is transversal to } H_{1}\right\}
$$

The first property of $\Delta_{W}$ is

Lemma 4.3.1. The collection $\Delta_{W}$ is non-empty.

For each $\delta$ in $\Delta_{W}$ we consider the open subset $\Gamma(\delta, W)$ of $\Gamma_{+}$given by

$$
\Gamma(\delta, W)=\left\{\gamma \in \Gamma_{+} \mid \gamma^{-1} \delta^{-1} W \quad \text { is transversal to } H_{1}\right\} .
$$

Let $R$ be the ring of analytic functions on $\Gamma(\delta, W)$ and let $\partial_{i \alpha}$ be the derivation of $R$ consisting of partial differentiation w.r.t. $t_{i \alpha}$. Then there holds

\section{Theorem 4.3.2.}

(a) For each $W \in \mathfrak{\wp}$ and each $\delta \in \Delta_{W}$ there is a unique function $\psi_{W}^{\delta}=\widehat{\psi}_{W}^{\delta} \cdot \delta$. $g(\lambda)$ of type $\delta$, such that $\psi_{W}^{\delta}(\gamma) \in W$ for all $\gamma \in \Gamma(\delta, W)$.

(b) The function $\psi_{W}^{\delta}$ from (a) is a wavefunction of type $\delta$. 
For a proof, we refer to [10]. If we write $\psi_{W}^{\delta}=K_{W}^{\delta} \cdot \delta \cdot g(\lambda)$, then we know from theorem 4.1.3 that

$$
L_{W}^{\delta}=K_{W}^{\delta} \partial\left(K_{W}^{\delta}\right)^{-1} \quad \text { and } \quad U_{\alpha, W}^{\delta}=K_{W}^{\delta} E_{\alpha}\left(K_{W}^{\delta}\right)^{-1}
$$

are solutions of the multicomponent KP-hierarchy. The following theorem makes clear why we did not consider the commuting flows from $\Gamma_{-}$.

Theorem 4.3.3. For each $g=\sum_{j \leq 0} \gamma_{j} \lambda^{j}$ in $\Gamma_{-}$, we have $L_{g W}^{\delta}=L_{W}^{\delta}$ and $U_{\alpha, g W}^{\delta}=$ $U_{\alpha, W}^{\delta}$

Remark 4.3.4. If $r>1$, then $\Delta_{W}$ may contain several elements. If $\delta_{1}$ and $\delta_{2}$ are in $\Delta_{W}$, then the solutions $\left\{L_{W}^{\delta_{1}}, U_{W, \alpha}^{\delta_{1}}\right\}$ and $\left\{L_{W}^{\delta_{2}}, U_{W, \alpha}^{\delta_{2}}\right\}$ are related by so-called differential difference equations that reduce in a specific case to the equations of the Toda-lattice, see [10]. These equations are a generalization to the KP-level of equations considered in [1].

In the one component case, one has the independent variables $t=\left\{t_{i} \mid i \geq 1\right\}$ and the derivatives $\left\{\partial_{i}=\frac{\partial}{\partial t_{i}} \mid i \geq 1\right\}$. One writes the elements of $R$ as functions in $t$. In [5], they introduced a new dependent variable called the $\tau$-function, that is related to a wavefunction of type 1 by

$$
\psi=\left\{1+\sum_{1>0} a_{i}(t) k^{-i}\right\} e^{\Sigma t_{i} k^{i}}=\frac{\tau\left(\left(t_{i}-\frac{1}{i k^{i}}\right)\right)}{\tau\left(\left(t_{i}\right)\right)} e^{\Sigma t_{i} k^{i}} .
$$

For $\tau$, depending polynomially of the $\left\{t_{i}\right\}$, a representation theoretic interpretation of the $\tau$-function was given in [15]. For a discussion of the role of the $\tau$-functions in the theory of inverse scattering, we refer the reader to [4].

It was shown in [21] how $\tau$ can be interpreted in the context of the line bundle $L((-1,0))$ over $\widetilde{\Im}^{(0)}$ and this gives an interpretation for a wider class of $\tau$ functions. In particular, these $\tau$-functions are Fredholm determinants of certain operators. Also in the multicomponent setting one can express the coefficients of $\widehat{\psi}_{W}^{\delta}$ in terms of Fredholm determinants related to the line bundle $L((-1,0))$. If $W=g_{1} F^{(0)}$, with $g_{1} \in G_{2}$, then we define $\tau_{g_{1} \mid H_{1}}: G \rightarrow \mathbb{C}$ by

$$
\tau_{g_{1} \mid H_{1}}((g, q))=(g, q) \cdot\left(f_{\Sigma_{1}}\right)\left(g_{1}\right)=\operatorname{det}\left(p_{S_{1}} \circ g^{-1} \circ\left(g_{1} \mid H_{1}\right) \circ q\right) .
$$

It measures the failure of $\widetilde{G}$-equivariance of the section corresponding to $f_{\Sigma_{1}}$. In $\Delta$ we consider the elements $\Delta_{i / j}$ given by

$$
\Delta_{i / j}=\operatorname{diag}\left(\ldots \lambda, \ldots, \lambda^{-1}, \ldots\right),
$$

where the $\lambda$-factor stands at the $i$-th place, the $\lambda^{-1}$-factor at the $j$-th place and the resulting factors are equal to 1 .

If $k \in \mathbb{C},|k|>1$, then we still need the element $q_{k}^{(i)}$ from $\Gamma_{+}$given by

$$
q_{k}^{(i)}=\operatorname{diag}\left(\ldots 1,1-\frac{\lambda}{k}, 1, \ldots\right),
$$


where the factor $1-\frac{\lambda}{k}$ stands at the $i$-th place. Then there holds

Theorem 4.3.5. Consider $a W$ in $\mathfrak{F}$ and $a$ in $\Delta_{W}$. Then $\delta^{-1}(W)=g \dot{F}^{(0)}$, with $g \in G_{2}$ and we have

(a) For all $1 \leq i \leq r$, the $(i, i)$-entry of $\widehat{\psi}_{W}^{\delta}$, is the $L^{2}$-boundary value of

$$
k \longmapsto \frac{\tau_{g \mid H_{1}}\left(\gamma q_{k}^{(i)}\right)}{\tau_{g \mid H_{1}}(\gamma)}
$$

(b) For $j \neq i$, there is a lifting $\widetilde{\Delta}_{i / j}$ of $\Delta_{i / j}$ to $G$ such that the $(j, i)$ entry of $\widehat{\psi}_{W}^{\delta}$ is the $L^{2}$-boundary value of

$$
k \longmapsto k^{-1} \frac{\tau_{g \mid H_{1}}\left(\gamma \widetilde{\Delta}_{i / j} q_{\xi}^{i}\right)}{\tau_{g \mid H_{1}}(\gamma)} .
$$

This theorem gives a geometric interpretation of formulae, stated in the appendix of [23]. For a proof of this theorem we refer the reader to [10]. There one can also find more equations that fit in the framework just described.

In the one component case the Japanese school, see [14], translates the equations that are satisfied by the wavefunction to equations for the $\tau$-function and these can be written in the so-called bilinear form

$$
\oint \tau\left(\left(t_{i}-\frac{1}{i k^{i}}\right)\right) \quad \tau\left(\left(s_{i}+\frac{1}{i k^{i}}\right)\right) e^{\sum_{i \geq 1}\left(t_{i}-s_{i}\right) k^{i}} d k=0,
$$

for all $\left(t_{i}\right)$ and $\left(s_{i}\right)$ and with $d k$ such that

$$
\oint \frac{d k}{2 \pi i k}=-1
$$

Also this formula can be given a geometric interpretation. For if $W \in \mathfrak{r}^{(0)}$ then one can consider $W^{\perp}$ as an element of the Grassmann manifold corresponding to $H=H_{2} \oplus H_{1}$. If $\psi_{W}$ is linked to $\tau$ by (10), then one can show

Theorem 4.3.6. The wavefunction $\psi_{W^{\perp}}$ can be expressed in $\tau$ by

$$
\widehat{\psi}_{W^{\perp}}(\lambda)=\lambda^{-1} \frac{\tau\left(\left(t_{i}+\frac{1}{i \lambda^{i}}\right)\right)}{\tau\left(\left(t_{i}\right)\right)}
$$

If we consider instead of $\Gamma_{+}$the group of flows consisting of the adjoints of the elements of $\Gamma_{+}$, then relation (11) boils down to the orthogonality relations for $\psi_{W}$ and $\psi_{W^{\perp}}$.

For $\ell \in \mathbb{Z}$, let $W_{\ell}$ be a general element of $\mathfrak{F}^{(\ell,-\ell)}$. According to the theorems 4.3.2 and 4.3.5 there corresponds a wavefunction of type $\lambda^{\ell}$ to $W_{\ell}$ and a $\tau$-function $\tau_{\ell}$ to $W_{\ell}$. In [14], they also discuss a system of non-linear equations that relates 2 different $\tau$-functions. For $\ell$ and $k \in \mathbb{Z}, k \geq \ell$, these equations are called the bilinear equations of the $(k, \ell)$-modified KP-hierarchy. They have the following interpretation in terms of the flag variety. 
Theorem 4.3.7. Let $k$ and $\ell \in \mathbb{Z}, k \geq \ell$, and let $W_{\ell}$ resp. $W_{k}$ belong to $\mathfrak{F}^{(\ell,-\ell)}$ resp. $\mathfrak{\Im}^{(k,-k)}$. If $W_{\ell} \supseteq W_{k}$, then $\tau_{\ell}$ and $\tau_{k}$ satisfy the equations of the $(k, \ell)$-modified $K P$ hierarchy.

For a proof we refer the reader to [12].

Remark 4.3.8. Hereby we have given a geometric description of the solutions of the modified hierarchies belonging to the Segal-Wilson class. This generalizes the description of the rational solutions in [16].

\section{References}

[1] M.J. Bergvelt and A.P.E. ten Kroode, Differential-difference AKNS equations and homogeneous Heisenberg algebras, J. of Math. Physics 28 (1987), 302.

[2] A.L. Carey and S.N.M. Ruijsenaars, On fermion gauge groups, current algebras and KacMoody algebras, Acta Applicandae Mathematicae 10 (1987), 1-86.

[3] A.L. Carey, C.A. Hurst and D.M. O'Brien, Automorphisms of the canonical anticommutation relations and index theory, Journal of Functional Analysis 48 (1982), 360-393.

[4] R. Caroll, Topics in soliton theory, North Holland, Amsterdam (1991).

[5] E. Date, M. Jimbo, M. Kashiwara, T. Miwa, Transformation groups for soliton equations, Proc. Japan Acad., 57A (1981), 342-7.

[6] A. Grothendieck, Sur la classification des fibrés holomorphes sur la sphère de Riemann, Am. J. Math., 79 (1957), 121-38.

[7] A. Grothendieck, La théorie de Fredholm, Bull. Soc. Math. France 84 (1956), 319-384.

[8] G.F. Helminck, Cylinder measures on Hilbert flag manifolds, to appear.

[9] G.F. Helminck and A.G. Helminck, The structure of Hilbert flag varieties, to appear.

[10] G.F. Helminck and G.F. Post, The geometry of differential difference equations, Memorandum 999, University of Twente (1991).

[11] G.F. Helminck, The Kähler structure of Hilbert flag varieties, to appear.

[12] G.F. Helminck and G.F. Post, Geometric interpretation of the bilinear equations for the KPhierarchy, Lett. Math. Phys. 16 (1988), 359-364.

[13] M. Hervé, Analytic and plurisubharmonic functions in finite and infinite dimensional spaces, Lecture Notes in Mathematics Vol. 198. Springer Verlag, Berlin (1971).

[14] M. Jimbo and T. Miwa, Solitons and infinite dimensional Lie algebras, Publication 439, RIMS, Kyoto (1983).

[15] V.G. Kac, Infinite dimensional Lie algebras, Birkhäuser, Boston (1983).

[16] V.G. Kac, D.H. Peterson, Lectures on the infinite wedge representation and the MKP hierarchy, Séminaire de Math. Sup. 102 (1986), Montreal University, 141-184.

[17] N.H. Kuiper, The homotopy type of the unitary group of Hilbert space, Topology 3 (1965), 19-30.

[18] J. Mickelsson, Current algebras and groups, Plenum monographs in nonlinear physics,

[19] D. Pickrell, Measures on infinite dimensional Grassmann manifolds, Journal of Functional Analysis 70 (1987), 323-356.

[20] A. Pressley and G. Segal, Loop groups, Clarendon Press, Oxford (1986).

[21] G.B. Segal and G. Wilson, Loop groups and equations of KdV-type, Publ. Math. I.H.E.S. 61 (1985), pp. 5-65.

[22] D. Shale, Linear symmetries of free Boson fields, Trans. Amer. Math. Soc. 103 (1962), 149167.

[23] K. Ueno and K. Takasaki, Toda-lattice hierarchy, Publication 423 RIMS, Kyoto, 1983. 
Depart ment of Mathematics, North Carol ina State University, Ral eigh, N.C., 276958205

E-mail address: loek@@cartan.math.ncsu.edu

Department of Mathemat ics, Universit eit Twente, Enschede, The Netherlands

E-mail address: helminck@@math . utwente.nl 